\title{
A CYBER-PHYSICAL SYSTEMS PAPER SURVEY ABOUT THE CONCEPT, ARCHITECTURE AND CHALLENGES FOR THE DEPLOYMENT WITHIN THE CONCEPT OF INDUSTRY 4.0
}

\author{
Markus MELICHER ${ }^{1}$, Dana ŠIŠMIŠOVÁ ${ }^{1}$, Ján VACHÁLEK ${ }^{1}$, \\ Cyril BELAVÝ ${ }^{1}$ \\ ${ }^{1}$ SLOVAK UNIVERSITY OF TECHNOLOGY IN BRATISLAVA \\ FACULTY OF MECHANICAL ENGINEERING \\ NÁM. SLOBODY 17, 81231 BRATISLAVA, SLOVAK REPUBLIC \\ e-mail: markus.melicher@stuba.sk, dana.sismisova@stuba.sk,jan.vachalek@stuba.sk, \\ cyril.belavy@stuba.sk \\ Received 19 August 2019, accepted 23 September 2019, published 29 November 2019
}

\begin{abstract}
The arrival of Cyber-physical systems provided space for a new emerging field of research oriented on this type of embedded systems. The aim of this paper is to provide a better understanding of this integrative research field focused on the concept, architecture and challenges in deployment of such systems within the concept of Industry 4.0. Cyber-physical systems represent an emerging area of research that attracts the interest of researchers around the world, because, in the field of design and development of future systems, they are expected to play a major role.
\end{abstract}

\section{Key words}

Cyber-physical systems, CPS, Industry 4.0, communications, Challenges

\section{INTRODUCTION}

Cyber-physical systems (CPSs) represented by the Chyba! Nenašiel sa žiaden zdroj odkazov., represent a significant evolutionary step in automation. They are physical devices with built-in tools for digital data collection, processing and distribution, connected with each other locally or remotely via the Internet [1]. 


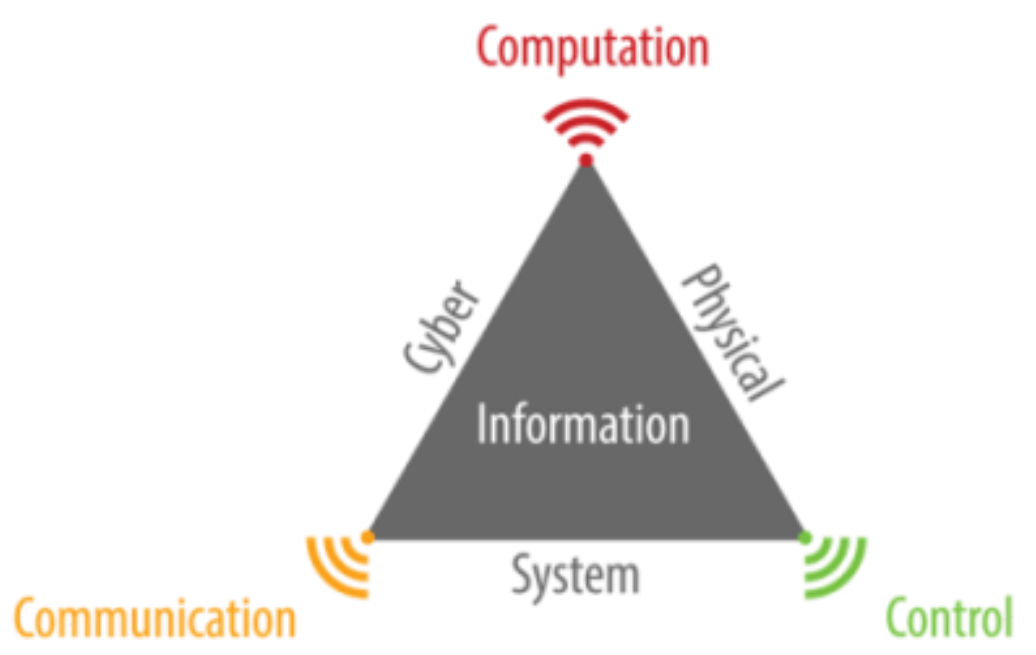

Figure 1 Ratio definition triangle between CPS [1]

CPS is the foundation for the Internet of Things, and, in combination with the Internet services, they form the foundation for Industry 4.0. The combination of CPS, high-performance software and special user interfaces that are integrated into digital networks opens a whole new world of the system functionality. Modern mobile phones are perhaps the most obvious example, offering an extensive package of applications and services that have completely changed the original principle of mobile phone operation [1], [1].

Cyber-physical systems are classified by generation as follows [1]:

Generation I - Contains identification technologies, such as RFID (Radio Frequency Identification) sensors, that allow unambiguous identification of the device they are built in.

Generation II - Are equipped with sensors and actuators with limited functionality.

Generation III - Can store, analyse and process data to a relatively large extent, and are equipped with multiple sensors and actuators.

CPS systems are an important element of manufacturing, products themselves, warehouse management, robotics, transport management, logistics services, supply chain management, fleet management, telemedicine and others. M2M (Machine to machine) Communication is a general name for communication over cyber-physical systems [1].

Nowadays, the trend in research and development is the area of internal platforms, which includes close integration of computing systems and physical systems, especially with regard to their control. The result of this trend is a new category of devices called cyber-physical systems. CPS systems can be simply described as the systems in which communication, control calculation and sensing are integrated. The aim of CPS systems is to built a close link between controlled physical processes and controlled computing systems [2].

\section{CYBER-PHYSICAL SYSTEMS AND INDUSTRY 4.0}

The previous three industrial revolutions were initiated by the arrival of technical innovations [2].

The first revolution was triggered by the arrival of steam engines. The second revolution occurred after the arrival of electricity and production lines. The third revolution was shaped by the boom in electrical engineering and information technology, which has led to industrial automation [2].

The Industry 4.0 initiative was launched at the fair in the German city of Hanover. The fourth revolution reflects customers' demands for cost-effectiveness and high production flexibility. Industry 4.0 uses cyber-physical systems, the Internet of Things and system services 
to meet the above requirements. Industry 4.0 allows for new business models, manufacturing concepts, intelligent control and tailored user requirements [2].

In today's production systems, constant adjustments are required to comply with the Industry 4.0 concept or model. For this reason, nested systems, consisting of control units, sensors and actuators, must have an Internet access interface. Such an interface can be achieved by extending the nested system to a cyber-physical system, and there may be several such extensions [2]:

- Direct System Expansion - This solution is a direct extension by connecting a communication interface, while the software adapts to network communication.

- Expansion of the system using a microcontroller-mounted expansion PCB - The system is connected to an optional microcontroller board, which contains a communication interface such as Ethernet, WiFi or CAN.

- Enhancement using smart sensors and actuators - Enhancement of this type is based on an architecture where communication to the Internet is controlled by smart sensors and actuators.

Cyber-physical systems are created by interconnecting into a larger and more complex set of systems to provide enhanced production control capabilities. Such integration of cyberphysical systems is also a major problem for efficient exploitation within the Industry 4.0 concept. Obstacles to integration are mainly due to semantic heterogeneity. Semantic heterogeneity is an ontology concept and represents an issue where database schemas or data sets from the same source are handled independently of two or more sources, the result of which may not be the same [2].

Everyday decisions within the company must be made with the least impact on their costs, but at the same time it is important to maintain not only the quality of the product, but also the quality of individual components, flexible human resources and other aspect involved in the production or process. A big advantage over competing companies has those companies that know their processes thoroughly and have enough information about them. Such companies produce very significant amounts of data per day, such as information about the machines, material, inventory, maintenance, planning, management, etc. [3].

\section{APLICATION OF CYBER-PHYSICAL SYSTEMS}

Cyber-physical systems are used in various areas, some of which may not have been originally designed for the integration of CPS systems. This includes, for example, aviation, transport, industry, healthcare and others. Some of the areas of application of CPS systems are briefly described in the following subsection [2], [4], [6]:

- Transportation - Transportation is becoming increasingly complex and requires progressively more complex systems. These integrated systems offer, for example, the ability to adjust vehicle behavior, entertainment factor, energy management and more. CPS systems are used in this area, for example in public transport, vehicle charging, traffic monitoring and driver support while driving.

- Medical and Health Systems - CPS in modern health systems are essential. Modern medicine is based on advanced technologies, where the emphasis is on high efficiency while minimizing side effects. CPS applications in the health sector include, for example, robotic operations, life support systems and others.

- Intelligent buildings - CPS find their application in the areas of monitoring and control of HVAC and electricity within buildings, applying the principle of feedback management. Sensor data gives feedback to the management to improve the safety, quality and environment of buildings. 
- Manufacturing - Because cyber-physical systems work on the principle of joining together, there are problems in manufacturing. Generally, the production sphere is a largely closed colossus, which largely does not reflect the latest technological knowledge in the field of computer science and automation. This closeness raises obstacles that make CPS not utilizing its potential. All processes and changes leading to higher integration of automation and CPS must be integrated in the business development plan in the long term, and the management must aim to meeting them. Common CPS integration in production carries the following features:

- A CPS collects data from the production process.

- The CPS evaluates the data collected and the production process.

$\circ \quad$ The CPS performs an action intervention in the production process.

There are also more complex applications in production:

- FESTO MiniProd system based on distributed control with the help of agentoriented architecture, while offering the possibility of programming and reprogramming on the fly.

- Model-driven production based on a 3D model robotic feedback control approach, enabling the operator to remotely control a physical robot at a remote location remotely using a cloud system.

\section{VARIATIONS OF CYBER-PHYSICAL SYSTEMS}

During the development of cyber-physical systems several iterations of different variants of CPS systems arose. The most specific types of CPS systems are described in the following subsection [2], [4], [5].

Specific one is the cyber-physical social system which relies on the integration of man and the CPS system. The common concept of a CPS system prefers separation of the human factor from the system. It follows that the physical, cyber and communication parts have been replaced by human knowledge, mental abilities and socio-cultural elements. A social CPS comprises an application where a human factor is required. Despite the superiority of cybernetic systems over humans, there are still applications where cybernetics cannot be fully utilized to increase automation, or it would be too costly. Another element is the social aspect where it is difficult to replace the human emotional approach with a machine [2], [4], [5].

Cloud cyber-physical systems are also one of many specific types of systems. These systems provide the ability to perform computational operations in a decentralized manner on significantly more powerful systems, and the corresponding results can be applied locally. Alternatively, it is necessary to combine data from several CPS systems in one place [2], [4], [5].

\section{THE CHALLENGES OF CYBER-PHYSICAL SYSTEMS}

Expectations regarding the cyber-physical systems are high. Mainly because of the robustness, self-organization, efficiency and interoperability of these systems, their implementation brings about many different challenges, depending on the field of application, motivated by expectations and requirements [2], [5].

The following list comprises some CPS systems challenges [2], [5]:

- Contextual systems - This area reflects the requirements for systems that are aware of the context of their implementation. Their functionality is important in terms of implementation of recognition, analysis and interpretation of CPS systems intentions.

- Cooperative production systems - Cyber-physical systems can create a complex interconnected structure, while the problem is not to create individual connections, but to achieve a uniform cooperation of CPS systems among themselves. 
- Prediction of dynamic systems - Expand available predictive methods or design new methods.

- Efficient and reliable systems - CPS systems can be assembled from many components (sensors, actuators and control units) manufactured by different manufacturers of individual components. Therefore, it is important to emphasize the proper composition of these components as well as thorough testing.

Research in the field of CPS is currently divided into several disciplines such as: communication and networking, system theory, mathematical calculations, software development, computer science and sensors. By deduction, it is possible to find that CPS systems are designed and analysed by means of several modelling tools and machinery. This gives rise to various representations that bring some functionality without checking the implications for others, which provides a more thorough analysis owing to their interdependence [6], [7].

\section{CONCLUSION}

The paper presents a short survey regarding the concept, architecture and challenges for the deployment of cyber-physical systems within the concept of industry 4.0, to which the leading countries pay nowadays more attention. Owing to the exponential evolution, we still encounter new problems and challenges in areas around the cyber-physical systems themselves. The study of state-of-the-art literature has challenged a need to develop cyber-physical systems for every of the various application scopes. Cyber-physical systems development is nowadays strongly supported by the current rapid technology advancement opening the opportunity to develop new prototypes and carry out research. Such new areas of research could also cause the immense speeding up of the evolution within the industry.

\section{Acknowledgement}

The authors thank the Slovak University of Technology, the Faculty of Mechanical Engineering, the Institute of Automation, Measurement and Applied Informatics and the Ministry of Education, Science, Research and Sport of the Slovak Republic. This article was written thanks to the subsidies from the state resources budget of the Ministry of Education, Science, Research and Sport of the Slovak Republic through the Project No. 002STU-2-1/2018 called STU as a leader of digital coalition.

\section{References}

[1] http://industry4.sk - Sova Group portal about the Industry 4.0 revolution [Online]. [Accessed: 032019] Available at http://industry4.sk.

[2] JIRKOVSKÝ, V. 2017. Semantic integration in the context of cyber-physical systems. Dissertation thesis.

[3] TANUSKA, P., SPENDLA, L., KEBISEK, M., VAZAN, P., HRCKA, L. 2017. Data Integration and Transformation Proposal for Big Data Analyses in Automotive Industry. In: 21 st IEEE International Conference on Intelligent Engineering Systems 2017 (INES 2017), Larnaca, pp. 97 102, ISBN 978-1-4799-7678-2.

[4] RAJKUMAR, R., LEE, I., SHA, L., STANKOVIC, J. 2010. Cyber-physical Systems: The Next Computing Revolution. In: 47th Design Automation Conference (DAC 2010), Anaheim, pp. 731736, ISBN 9781-4-5030-002-5.

[5] LEE, E. A., SESHIA, S. A. 2016. Introduction to embedded systems: A cyber-physical systems approach. MIT Press, ISBN 978-0-262-53381-2. 
[6] SHI, J., WAN, J., YAN, H., SUO, H. 2011. A Survey of Cyber Physical Systems. In: Proc. of the Int. Conf. on Wireless Communications and Signal Processing (WCSP 2011), Nanjing, ISBN 9781-4577-1010-0.

[7] SANISLAV, T., MICLEA, L. 2012. Cyber-Physical Systems - Concept, Challenges and Research Areas. In: Control Engineering and Applied Informatics (CEAI 2012), Romania, No.2, pp. 28-33.

\section{ORCID}

Markus Melicher

0000-0003-0020-8808 